\title{
Mean Performance of Normal and QPM Maize Genotypes for Yield and Tryptophan Content
}

\author{
M. Surender ${ }^{1 *}$, Deepti Sagare ${ }^{1}$, Prashant Setti ${ }^{1}$, Ch.V. Durga Rani ${ }^{1}$, \\ Farzana Jabeen ${ }^{1}$, M.R. Sudarshan ${ }^{2}$ and S. Sokka Reddy ${ }^{1}$ \\ ${ }^{1}$ Institute of Biotechnology, Professor Jayashankar Telangana State Agricultural University, \\ Hyderabad-30, Telangana, India \\ ${ }^{2} \mathrm{MRC}, \mathrm{ARI}$, Rajendranagar, Telangana, India \\ *Corresponding author
}

\begin{tabular}{|c|c|}
\hline & A B S T R A C T \\
\hline & \multirow{5}{*}{$\begin{array}{l}\text { An investigation was taken up at Maize Research Station, Agricultural Research Institute, } \\
\text { PJTSAU, Hyderabad during } 2013-14 . \mathrm{F}_{1} \text { material was generated by making crosses } \\
\text { between BML } 6 \times \text { CML } 181 \text { and BML } 7 \times \text { CML } 181 \text { for introgression of opaque } 2 \text { gene } \\
\text { from Mexican donor CML181 through molecular breeding approach. The converted } \\
\text { quality protein maize BML } 6 \text { and BML } 7 \text { lines (designated as CB6 and CB7) with } \\
\text { increased levels of lysine and tryptophan were crossed in various combinations to select a } \\
\text { hybrid with increased lysine, tryptophan and high yield. The performance of elite maize } \\
\text { genotypes, donor and derived QPM inbreds and their hybrid combinations were analysed } \\
\text { for yield and yield attributing characters. The experiment was designed with three } \\
\text { replication following Randomized Block Design. Observations were recorded on twelve } \\
\text { quantitative and qualitative traits. Hybrids recorded superior performance to parents with } \\
\text { respect to plant height (cm), ear height (cm), number of kernel rows per ear, } 100 \text { kernel } \\
\text { weights (g), grain yield per plant (g) and tryptophan content }(\%) \text {. Two cross combinations } \\
\text { CB6-36 } \times \text { CB7-28 and CB6-36 } \times \text { CB7-59 exhibited relatively higher tryptophan content } \\
\text { and on par grain yield per plant compared to the standard check DHM } 117 \text {. The hybrid } \\
\text { combination CB6-36 } \times \text { CB7-28 was shown excellent performance at All Indian Coordinate } \\
\text { Research Project (AICRP) on Maize trails during the year } 2015-16 \text { and the yield was } 6924 \\
\text { Kg/ha (BQPMH141) with higher tryptophan content and these hybrid parental lines need } \\
\text { to be further multiplied for commercial exploitation. }\end{array}$} \\
\hline Keywords & \\
\hline Article Info & \\
\hline $\begin{array}{l}\text { Accepted: } \\
10 \text { September } 2017 \\
\text { Available Online: } \\
10 \text { November } 2017\end{array}$ & \\
\hline & \\
\hline
\end{tabular}

\section{Introduction}

Maize supplies up to 60 percent of the daily human protein (Sofi et al., 2009). However protein content of normal maize lacks two important amino acids namely, lysine and tryptophan that are nutritionally essential for humans (Krivanek et al., 2007; Sofi et al., 2009 and Mbuya et al., 2011). The QPM research was initiated long back during 1970's but it gained momentum during 1990's with continuous breeding efforts on development of high yielding hard endosperm modified opaque2 maize germplasm by International Centre for Maize and Wheat improvement (CIMMYT) which made it available for use in the breeding programme all over the world. In India germplasm 
accessions received from CIMMYT, Mexico were tested at different centers of All India Coordinated Research Project (AICRP) on Maize. Through acclimatization and repeated selection, hard endosperm modified opaque 2 maize inbred lines and their crosses have been identified for better protein quality and higher yield potential. In 1997, Indian Institute of Maize Research (IIMR), New Delhi developed first QPM composite variety Shakti-1 with $0.63 \%$ tryptophan, which was released and recommended for general cultivation in Indian during 1998. The lines were evaluated for their productivity and deployed in combination breeding which led to release of first QPM three way cross hybrid Shaktiman-1 by Rajendra Agricultural University (RAU), Pusa during 2001 followed by release of first QPM single cross hybrid Shaktiman-2 during 2004 for their cultivation in Bihar state. In 2005 Chaudhary Charan Sing Haryana Agricultural University (CCSHAU), Karnal released another QPM single cross hybrid HQPM-1 which is the first yellow grain QPM single cross hybrid released for its cultivation across the country. Later in series of QPM, Shaktiman-3 and Shaktiman-4 were released by RAU, Pusa during 2006 for their cultivation in Bihar. In 2007 another QPM single cross hybrid HQPM-5 from CCHSAU, Karnal and Vivek QPM-9 from Vivekanand Parvathiya Krishi Anusandhan Sanstan (VPKAS), Almora have been identified during 2008. Vivek QPM-9 has a unique distinction over the QPM hybrids as the first marker assisted selection product of normal hybrid Vivek-9 (Gupta et $a l ., 2013)$. The current study deals with the conversion of non QPM inbred lines to QPM with more than $4 \%$ lysine and $0.95 \%$ tryptophan.

\section{Materials and Methods}

The present investigation was carried out during Kharif 2011 to Rabi 2014-15 at Maize
Research Centre, Agricultural Research Institute and Institute of Biotechnology, Rajendranagar, Hyderabad. The Quality Protein Maize (QPM) germplasm and non QPM lines for the present investigation were obtained from Indian Institute of Maize Research (IIMR), New Delhi, Maize Research Centre (MRC), Rajendranagar, Hyderabad and Vivekananda Parvatiya Krishi Anusandhan Sansthan (VPKAS), Almora. Germplasm line was shown in the table 1.

Observations were recorded on days to 50 percent tasseling, days to 50 percent silking, days to maturity, plant height $(\mathrm{cm})$, ear height $(\mathrm{cm})$, ear girth $(\mathrm{cm})$, ear length $(\mathrm{cm})$, number of kernel rows per ear, number of kernels per row, 100 seed weight (g), grain yield per plant (g) and tryptophan content $(\%) . \mathrm{F}_{1}$ material was generated by making crosses between BML $6 \times$ CML 181 and BML $7 \times$ CML 181 . Two $\mathrm{F}_{1}$ populations $(\mathrm{BML} 6 \times \mathrm{CML} 181$ and BML $7 \times$ CML 181) were back crossed with respective recurrent parents to generate $\mathrm{BC}_{1} \mathrm{~F}_{1}$ material. After foreground and background selection $\mathrm{BC}_{1} \mathrm{~F}_{1}$ material with high $\mathrm{RPG}$ was again back crossed with respective recurrent parents to generate $\mathrm{BC}_{2} \mathrm{~F}_{1}$ population. These crosses were affected during Kharif 2011 and Rabi 2011-12. Foreground selection and light box analysis was performed in $\mathrm{BC}_{2} \mathrm{~F}_{2}$ population. Seeds showing $<25 \%$ opacity were analyzed for tryptophan estimation and high tryptophan containing lines was selfed to generate $\mathrm{BC}_{2} \mathrm{~F}_{4}$ population during Rabi 201213. The converted lines of BML 6 and BMIL 7 in $\mathrm{BC}_{2} \mathrm{~F}_{4}$ were designated as CB6 and CB7, respectively. The selected converted inbreds were used for crossing program.

The converted BML 6 and BML 7 lines with increased levels of lysine and tryptophan were crossed in various combinations to select a hybrid with increased lysine, tryptophan and high yield. Inbreds used in the present study were given in the table 1 . The analysis of 
variance for each character was carried out by using the method described by Panse and Sukhatme (1985). The test of significance was carried out by referring to ' $F$ ' table values given by Fisher and Yates (1967).

\section{Results and Discussion}

The converted BML6 and BML7 were designated as $\mathrm{CB} 6$ and $\mathrm{CB} 7$ with their pedigree plant numbers. The selected families were used for crossing program for producing QPM hybrid. The stable expression of these traits was confirmed through $\mathrm{BC}_{2} \mathrm{~F}_{3}$ families, which represented the final converted QPM versions of the original recipient lines.

The expression of genetic modifiers may be affected by maternal influence. Endosperm is a triploid tissue; one may expect maternal influence since two doses of modifying alleles are contributed by the maternal parent and only one by the paternal parent.

Reciprocal differences in crosses between soft opaque and modified opaque have been reported by Vasal, 1994. Other factors such as genetic background and kernel texture can also alter phenotypic manifestation of modifying genes. Flint genetic backgrounds generally exhibit a higher frequency of modified kernels (Vasal, 1994). Therefore, direct and reciprocal crosses between selected families of CB6 and CB7 inbreds were performed to generate $\mathrm{F}_{1}$ hybrids.

In direct cross, CB6-36, CB6-39 as female parent and CB7-11, CB7-28 and CB7-59 as male parent and in reciprocal crosses it was vice-versa for producing QPM hybrid. The six $\mathrm{F}_{1}$ hybrids were evaluated during the Rabi 2013-14 and Kharif 2014 along with five converted inbreds, two normal inbred, one QPM inbred, one standard check hybrid DHM117 and one standard reciprocal check hybrid BML7 X BML6 (Table 2).

\section{Evaluation of QPM version of DHM117 hybrid}

During Rabi 2013-14 and Kharif 2014, all crosses (Table 2) along with 5 parents, 3 check inbreds (BML6, BML7 and CML181) one commercial check, DHM117 and reciprocal cross between BML7 X BML6 were sown in Randomized Block Design (RBD) replicated thrice. Each entry was sown in a row of five meter length with a spacing of $75 \mathrm{~cm}$ between rows and $20 \mathrm{~cm}$ between the plants. Necessary plant protection measures were taken to protect the crop from pests and diseases as per the Maize Research Center recommendations, so as to raise a healthy crop.

Converted inbreds (CB6, CB7) and QPM hybrid purity assessment was checked in Rabi 2013-14 using gene specific umc1066 SSR marker. All the QPM hybrids possessed recessive allele of opaque2. Phenotypic data was recorded on twelve different characters viz., days to 50 per cent tasseling, days to 50 per cent silking, days to maturity, plant height $(\mathrm{cm})$, ear height $(\mathrm{cm})$, ear length $(\mathrm{cm})$, ear girth $(\mathrm{cm})$, number of kernel rows per ear, number of kernels per row, 100 kernel weights (g), grain yield per plant (g) and tryptophan content $(\%)$ in generated hybrids.

The data was recorded on twelve different characters viz., days to 50 per cent tasseling, days to 50 per cent silking, days to maturity, plant height $(\mathrm{cm})$, ear height $(\mathrm{cm})$, ear length $(\mathrm{cm})$, ear girth $(\mathrm{cm})$, number of kernel rows per ear, number of kernels per row, 100 kernel weights $(\mathrm{g})$, grain yield per plant $(\mathrm{g})$ and tryptophan content (\%).

The analysis of variance of the parents and the hybrids for yield and yield attributing characters are presented in Table 3. The mean sum of squares for genotypes (parents and hybrids) was highly significant for all the 
traits studied except for days to maturity and tryptophan content indicating the existence of sufficient variability in the material studied (Fisher and Yates, 1967).

The mean performance of parents, hybrids and standard checks for the twelve characters studied during Rabi 2013-14 and Kharif 2014 are presented in Table 2 to 9.

\section{Days to 50 percent tasseling}

For days to 50 percent tasseling the mean values ranged from 66.47 (CB6-36) to 72.01 (CB7-59) days for converted lines, 69.35 (BML 6) to 71.80 (BML 7) days for testers and 64.73 (CB6-36 $\times$ CB7-11) to 71.70 (CB7$39 \times$ CB7-59) days for QPM hybrids (Table 2). In case of checks, DHM 117 and reciprocal cross BML7 $\times$ BML 6 recorded 70.63 and 71.12 days respectively. Among converted lines, 66.47 (CB6-36) was earliest and among testers, BML 6 (69.35) was earliest for days to 50 percent tasseling. Hybrid CB6-36 × CB7-11 was earliest while CB7-11 $\times$ CB6-36, CB7-11 $\times$ CB6-39 and CB6-36 $\times$ CB7 -28 were on par with CB6-36 $\times$ CB7- 11 and significantly superior to others. Over the standard check DHM117 (70.63), 8 hybrids were found to be significantly superior for earliness.

\section{Days to 50 percent silking}

The mean values for days to 50 percent silking ranged from $68.87(\mathrm{CB}-36)$ to 74.60 (CB7-59) days for converted lines, 73.43 (BML 6) to 74.32 (BML 7) days for testers and 67.79 (CB6-36 × CB7-11) to 75.13 (CB6$39 \times$ CB7-59) days for QPM hybrids. Checks DHM 117 and reciprocal cross BML7 $\times$ BML 6 recorded 72.98 and 73.82 days respectively. Line CB6-36 (68.87) was earliest and significantly superior to the remaining lines (Table 2). Among testers, BML 6 (73.43) was earliest and significant. The hybrid, CB6-36 $\times$
CB7-11(67.79) was earliest while others (CB7-11 × CB6- 36 and CB6-36 × CB7-28) were on par with the QPM hybrid CB6-36 $\times$ CB7- 11 and significantly superior. When compared to standard check DHM 117 (72.98), 7 hybrids were significantly superior. The results are in agreement with earlier findings of Maqsood et al., (2007).

\section{Days to maturity}

The mean values for this trait ranged from 111.67 (CB6-36) to 115.87 (CB7-59) days for converted lines, 111.13 (BML 6) to 113.10 (BML7) days for testers and 107.45 (CB7-28 $\times$ CB6-36) to $111.20(\mathrm{CB} 7-59 \times \mathrm{CB} 6-36)$ days for QPM hybrids. In case of checks, DHM 117 and BML $7 \times$ BML 6 recorded 113.30 and 114.17 days respectively. Among converted lines CB6-36 (111.67) was earliest and significantly superior to the remaining lines. Among testers, BML6 (111.13) was earliest and significantly superior to the remaining testers. Among the QPM hybrids, CB7-28 × CB6-36 (107.45) was earliest and remaining hybrids were on par with the hybrid CB7-28 × CB6-36. Over the standard check DHM117 (113.3), 12 hybrids were significantly superior for earliness (Table 3). Similar types of results were reported by Yusuf (2013).

\section{Plant height (cm)}

The mean values for plant height ranged from 135.37 (CB7-11) to $161.42 \mathrm{~cm}$ (CB7-59) for converted lines, 153.55 (BML 6) to 169.57 $\mathrm{cm}(\mathrm{BML} 7)$ for testers and 196.05 (CB6-39 $\times$ CB7- 59 to $223.14 \mathrm{~cm}(\mathrm{CB} 7-28 \times \mathrm{CB} 6-36)$ for hybrids (Table 3). Hybrids recorded higher average height $(206.24 .15 \mathrm{~cm})$ compared to lines $(152.09 \mathrm{~cm})$ and testers $(152.83 \mathrm{~cm})$. In case of standard checks DHM 117 and reciprocal BML $7 \times$ BML 6 recorded 211.98 and $213.3 \mathrm{~cm}$ of plant height respectively. Among lines CB7-59 recorded 
maximum plant height $(161.42 \mathrm{~cm})$ to the remaining lines. Among testers, BML 7 recorded maximum plant height $(169.57 \mathrm{~cm})$ and superior to remaining testers. Among hybrids, maximum plant height was recorded for CB7-28 $\times$ CB6-36 $(223.14 \mathrm{~cm})$ and two hybrids CB7-59 $\times$ CB6-39 and CB7-11 $\times$ CB6- 39 were on par with the best hybrid CB7-28 × CB6-36 (223.14cm). Yusuf (2013) reported similar kind or results.

\section{Ear height (cm)}

Mean values for ear height ranged from 50.63 (CB7-59) to $74.11 \mathrm{~cm}$ (CB6-36) for converted lines, 60.20 (BML6) to $62.00 \mathrm{~cm}$ (BML 7) for testers and $75.09(\mathrm{CB} 6-36 \times \mathrm{CB} 7-28)$ to 99.52 cm (CB7-11 × CB6-39) for hybrids. Hybrids recorded greater average height $(87.76 \mathrm{~cm})$ compared to lines $(64.87 \mathrm{~cm})$ and testers $(57.55 \mathrm{~cm})$. In case of checks, DHM 117 and reciprocal BML7 $\times$ BML6 recorded 97.41 and $98.39 \mathrm{~cm}$ of ear height respectively (Table 4 ). Among the converted lines, CB6-36 (74.11 $\mathrm{cm})$ recorded maximum ear height to other lines. Among the testers, BML7 $(62.00 \mathrm{~cm})$ recorded maximum ear height and significantly superior to other testers. One hybrid (CB6-36 × CB7-28) was significantly superior to the remaining hybrids for this trait. When compared with standard check DHM 117 , almost all hybrids were on par with it for ear height. The present findings are similar to those reported by Yusuf (2013).

Table.1 Inbreds used in the present study and their pedigree

\begin{tabular}{|c|c|c|}
\hline S.No & Inbreds & pedigree \\
\hline 1 & CB6-36 & \multirow[b]{2}{*}{ Derived QPM inbred from cross between BML6 and CML 181} \\
\hline 2 & CB6-39 & \\
\hline 3 & CB7-11 & \multirow{3}{*}{ Derived QPM inbred from cross between BML 7 and CML 181} \\
\hline 4 & CB7-28 & \\
\hline 5 & CB7-59 & \\
\hline 6 & BML6 & \multirow[b]{2}{*}{ Recurrent parents derived from foreign germplasm } \\
\hline 7 & BML7 & \\
\hline 8 & CML181 & Mexican donor \\
\hline 9 & DHM117 & $\begin{array}{l}\text { Popular single cross hybrid used as a Standard check derived from } \\
\text { cross between BML6 and BML } 7\end{array}$ \\
\hline
\end{tabular}

Table.2 Crosses attempted for making direct and reciprocal hybrids

\begin{tabular}{|c|l|l|l|}
\hline S.No & \multicolumn{1}{|c|}{ Direct } & \multicolumn{1}{|c|}{ S.No } & \multicolumn{1}{c|}{ Reciprocal } \\
\hline 1 & CB6-36 X CB7-28 & 1 & CB7-11 XCB6-36 \\
\hline 2 & CB6-36 X CB7-28 & 2 & CB7-11 XCB6-39 \\
\hline 3 & CB6-36 X CB7-59 & 3 & CB7-28 X CB6-36 \\
\hline 4 & CB6-39 X CB7-11 & 4 & CB7-28 X CB6-39 \\
\hline 5 & CB-39 X CB7-28 & 5 & CB7-59 XCB6-36 \\
\hline 6 & CB-39 X CB7-59 & 6 & CB7-59 XCB6-39 \\
\hline Check & DHM117 (BML6 X BML7) & Check & BML7 X BML6 \\
\hline
\end{tabular}


Table.3 Analysis of variance for randomized block design for yield and yield attributing characters in maize

\begin{tabular}{|c|c|c|c|}
\hline \multirow{2}{*}{ Character } & \multicolumn{2}{|c|}{ Mean sum of square } \\
\cline { 2 - 4 } & $\begin{array}{c}\text { Replication } \\
(\mathbf{d f}=\mathbf{2})\end{array}$ & $\begin{array}{c}\text { Genotypes } \\
(\mathbf{d f}=\mathbf{2 1})\end{array}$ & $\begin{array}{c}\text { Error } \\
(\mathbf{d f}=\mathbf{4 2})\end{array}$ \\
\hline Days to 50\% tasseling & 0.154 & $27.80^{* *}$ & 0.62 \\
\hline Days to 50\% silking & 0.466 & $25.93^{* *}$ & 0.58 \\
\hline Days to maturity & $9.577^{* *}$ & $11.43^{* *}$ & 1.27 \\
\hline Plant height (cm) & 339.581 & $2923.94^{* *}$ & 351.49 \\
\hline Ear height (cm) & 0.009 & $713.49^{* *}$ & 2.10 \\
\hline Ear length (cm) & 0.063 & $18.68^{* *}$ & 0.033 \\
\hline Ear girth (cm) & 0.031 & $9.84^{* *}$ & 0.024 \\
\hline Number of kernel rows per ear & 0.016 & $2.18^{* *}$ & 0.025 \\
\hline Number of kernels per row & 0.329 & $205.48^{* *}$ & 0.48 \\
\hline 100 kernel weight (g) & 0.44 & $178.39^{* *}$ & 0.14 \\
\hline Grain yield per plant (g) & 57.041 & $5604.50^{* *}$ & 3.16 \\
\hline Tryptophan content (\%) & $0.001^{* *}$ & $0.07^{* *}$ & 0.00 \\
\hline
\end{tabular}

*significant at $5 \%$ level; ** significant at $1 \%$ level

Table.4 Mean performance of parents, crosses and standard checks for days to 50 percent tasseling and days to 50 percent silking in two seasons

\begin{tabular}{|c|c|c|c|c|c|c|}
\hline Source & $\begin{array}{c}\text { Rabi 2013-14 } \\
\text { Days to 50\% } \\
\text { tasseling } \\
\end{array}$ & $\begin{array}{c}\text { Kharif-2014 } \\
\text { Days to } 50 \% \\
\text { tasseling } \\
\end{array}$ & mean & $\begin{array}{c}\text { Rabi 2013-14 } \\
\text { Days to 50\% } \\
\text { silking } \\
\end{array}$ & $\begin{array}{c}\text { Kharif2014 } \\
\text { Days to } 50 \% \\
\text { silking } \\
\end{array}$ & mean \\
\hline CB6-36 & 66.41 & 66.53 & 66.47 & 68.81 & 68.93 & 68.87 \\
\hline CB6-39 & 70.27 & 70.67 & 70.47 & 72.67 & 73.52 & 73.10 \\
\hline CB7-11 & 71.17 & 71.82 & 71.50 & 74.60 & 74.60 & 74.60 \\
\hline CB7-28 & 70.69 & 70.52 & 70.61 & 73.53 & 73.75 & 73.64 \\
\hline CB7-59 & 72.28 & 71.74 & 72.01 & 74.70 & 74.21 & 74.46 \\
\hline Lines mean & 70.16 & 70.26 & 70.21 & 72.86 & 73.00 & 72.93 \\
\hline Lines range & 66.41 to 72.28 & 66.53 to 71.82 & 66.47 to 72.01 & 68.81 to 74.70 & 68.93 to 74.60 & 68.87 to 74.60 \\
\hline BML6 & 69.70 & 69.00 & 69.35 & 72.90 & 73.97 & 73.43 \\
\hline BML7 & 71.93 & 71.67 & 71.80 & 74.43 & 74.20 & 74.32 \\
\hline CML181 & 79.42 & 79.60 & 79.51 & 82.46 & 82.57 & 82.51 \\
\hline Checks mean & 73.68 & 73.42 & 73.55 & 76.60 & 76.91 & 76.75 \\
\hline Checks range & 69.70 to 79.42 & 69.00 to 79.60 & 69.35 to 79.51 & 72.90 to 82.46 & 73.97 to 82.57 & 73.43 to 82.51 \\
\hline CB6-36×CB7-11 & 64.52 & 64.93 & 64.73 & 67.40 & 68.17 & 67.79 \\
\hline CB6-36×CB7-28 & 65.92 & 67.30 & 66.61 & 68.40 & 69.84 & 69.12 \\
\hline CB6-36×CB7-59 & 70.00 & 71.67 & 70.83 & 73.10 & 74.60 & 73.85 \\
\hline CB6-39×CB7-11 & 67.60 & 68.20 & 67.90 & 71.30 & 71.97 & 71.63 \\
\hline CB6-39×CB7-28 & 70.00 & 69.34 & 69.67 & 72.83 & 72.43 & 72.63 \\
\hline CB6-39×CB7-59 & 71.84 & 71.55 & 71.70 & 75.80 & 74.47 & 75.13 \\
\hline CB7-11×CB6-36 & 64.46 & 65.90 & 65.18 & 67.93 & 68.90 & 68.42 \\
\hline CB7-11×CB6-39 & 65.55 & 66.37 & 65.96 & 68.50 & 70.51 & 69.51 \\
\hline CB7-28×CB6-36 & 70.16 & 69.57 & 69.87 & 72.90 & 72.16 & 72.53 \\
\hline CB7-28×CB6-39 & 69.50 & 70.02 & 69.76 & 73.40 & 72.40 & 72.90 \\
\hline CB7-59×CB6-36 & 70.93 & 70.39 & 70.66 & 74.46 & 72.77 & 73.61 \\
\hline CB7-59×CB6-39 & 71.16 & 71.85 & 71.51 & 73.83 & 73.96 & 73.89 \\
\hline Hybrids mean & 68.47 & 68.92 & 68.70 & 71.65 & 71.85 & 71.75 \\
\hline Hybrids range & 64.46 to 71.84 & 64.93 to 71.85 & 64.73 to 71.70 & 67.40 to 75.80 & 68.17 to 74.60 & 67.79 to 75.13 \\
\hline \multicolumn{7}{|l|}{ Check } \\
\hline DHM-117 & 70.10 & 71.16 & 70.63 & 72.43 & 73.53 & 72.98 \\
\hline BML7×BML6 & 70.76 & 71.63 & 71.195 & 73.43 & 74.20 & 73.815 \\
\hline checks mean & 70.43 & 71.40 & 70.91 & 72.93 & 73.87 & 73.40 \\
\hline checks range & 70.10 to 70.76 & 71.16 to 71.63 & 70.63 to 71.19 & 72.43 to 73.43 & 73.53 to 74.20 & 72.98 to 73.81 \\
\hline $\mathrm{CD}$ & 1.967 & 1.301 & 1.634 & 1.724 & 1.257 & 1.4905 \\
\hline $\mathrm{SE}(\mathrm{d})$ & 0.971 & 0.642 & 0.8065 & 0.851 & 0.621 & 0.736 \\
\hline $\mathrm{SE}(\mathrm{m})$ & 0.687 & 0.454 & 0.5705 & 0.602 & 0.439 & 0.5205 \\
\hline CV & 1.705 & 1.123 & 1.414 & 1.434 & 1.042 & 1.238 \\
\hline
\end{tabular}


Table.5 Mean performance of parents, crosses and standard checks for days to maturity and plant height in two seasons

\begin{tabular}{|c|c|c|c|c|c|c|}
\hline source & $\begin{array}{c}\text { Rabi2013-14 } \\
\text { days to } \\
\text { maturity }\end{array}$ & $\begin{array}{c}\text { Kharif-2014 } \\
\text { days to } \\
\text { maturity }\end{array}$ & mean & $\begin{array}{l}\text { Rabi 2013-14 } \\
\text { plant height } \\
\qquad(\mathrm{cm})\end{array}$ & $\begin{array}{l}\text { Kharif2014 } \\
\text { Plant height } \\
\text { (cm) }\end{array}$ & $\begin{array}{l}\text { Mean } \\
(\mathrm{cm})\end{array}$ \\
\hline CB6-36 & 112.33 & 111.00 & 111.67 & 159.63 & 159.03 & 159.33 \\
\hline CB6-39 & 112.63 & 111.57 & 112.10 & 144.50 & 143.60 & 144.05 \\
\hline CB7-11 & 113.46 & 112.53 & 113.00 & 159.83 & 110.90 & 135.37 \\
\hline CB7-28 & 113.60 & 111.87 & 112.73 & 160.86 & 159.73 & 160.30 \\
\hline CB7-59 & 116.67 & 115.07 & 115.87 & 163.40 & 159.43 & 161.42 \\
\hline Lines mean & 113.74 & 112.41 & 113.07 & 157.64 & 146.54 & 152.09 \\
\hline Lines range & 112.33 to 116.67 & $\begin{array}{c}111.00 \text { to } \\
115.07\end{array}$ & $\begin{array}{c}111.67 \text { to } \\
115.87\end{array}$ & $\begin{array}{c}144.50 \text { to } \\
163.40\end{array}$ & $\begin{array}{c}110.90 \text { to } \\
159.73\end{array}$ & $\begin{array}{c}135.37 \text { to } \\
161.42\end{array}$ \\
\hline BML6 & 110.87 & 111.40 & 111.13 & 154.04 & 153.07 & 153.55 \\
\hline BML7 & 113.27 & 112.93 & 113.10 & 169.16 & 169.98 & 169.57 \\
\hline CML181 & 114.80 & 114.83 & 114.82 & 135.26 & 135.47 & 135.36 \\
\hline checks mean & 112.98 & 113.05 & 113.02 & 152.82 & 152.84 & 152.83 \\
\hline Checks range & 110.87 to 114.80 & $\begin{array}{c}111.40 \text { to } \\
114.83\end{array}$ & $\begin{array}{c}111.13 \text { to } \\
114.82\end{array}$ & $\begin{array}{c}135.26 \text { to } \\
169.16\end{array}$ & $\begin{array}{c}135.47 \text { to } \\
169.98\end{array}$ & $\begin{array}{c}135.36 \text { to } \\
169.57\end{array}$ \\
\hline CB6-36×CB7-11 & 111.40 & 109.60 & 110.50 & 203.54 & 201.67 & 202.60 \\
\hline CB6-36×CB7-28 & 111.17 & 109.56 & 110.36 & 201.50 & 200.74 & 201.12 \\
\hline CB6-36×CB7-59 & 110.43 & 111.50 & 110.97 & 204.36 & 201.85 & 203.11 \\
\hline CB6-39×CB7-11 & 110.76 & 110.97 & 110.86 & 198.66 & 199.07 & 198.87 \\
\hline CB6-39×CB7-28 & 107.76 & 108.73 & 108.25 & 201.15 & 202.85 & 202.00 \\
\hline CB6-39×CB7-59 & 107.80 & 110.90 & 109.35 & 194.46 & 197.64 & 196.05 \\
\hline CB7-11×CB6-36 & 108.80 & 111.07 & 109.93 & 203.88 & 206.33 & 205.11 \\
\hline CB7-11×CB6-39 & 105.50 & 109.73 & 107.62 & 217.16 & 215.57 & 216.37 \\
\hline CB7-28×CB6-36 & 106.83 & 108.07 & 107.45 & 224.96 & 221.31 & 223.14 \\
\hline CB7-28×CB6-39 & 109.63 & 108.70 & 109.17 & 205.80 & 203.60 & 204.70 \\
\hline CB7-59×CB6-36 & 112.23 & 110.17 & 111.20 & 202.10 & 203.12 & 202.61 \\
\hline CB7-59×CB6-39 & 112.67 & 109.67 & 111.17 & 219.86 & 218.57 & 219.21 \\
\hline Hybrids mean & 109.58 & 109.89 & 109.74 & 206.45 & 206.03 & 206.24 \\
\hline Hybrids range & 105.50 to 112.67 & $\begin{array}{c}108.07 \text { to } \\
111.50\end{array}$ & $\begin{array}{c}107.45 \text { to } \\
111.20\end{array}$ & $\begin{array}{c}194.46 \text { to } \\
224.96\end{array}$ & $\begin{array}{c}197.64 \text { to } \\
221.31\end{array}$ & $\begin{array}{c}196.05 \text { to } \\
223.14\end{array}$ \\
\hline \multicolumn{7}{|l|}{ check } \\
\hline DHM-117 & 112.93 & 113.30 & 113.30 & 210.54 & 213.43 & 211.98 \\
\hline BML7×BML6 & 113.83 & 114.17 & 114.17 & 213.06 & 213.53 & 213.29 \\
\hline checks mean & 113.38 & 113.74 & 113.74 & 211.80 & 213.48 & 212.64 \\
\hline checks range & 112.93 to 113.83 & $\begin{array}{c}113.30 \text { to } \\
114.17\end{array}$ & $\begin{array}{c}113.30 \text { to } \\
114.17\end{array}$ & $\begin{array}{c}210.54 \text { to } \\
213.06\end{array}$ & $\begin{array}{c}213.43 \text { to } \\
213.53\end{array}$ & $\begin{array}{c}211.98 \text { to } \\
213.29\end{array}$ \\
\hline $\mathrm{CD}$ & 1.451 & 1.86 & 1.6555 & 2.576 & 31.001 & 16.7885 \\
\hline $\mathrm{SE}(\mathrm{d})$ & 0.716 & 0.919 & 0.8175 & 1.272 & 15.308 & 8.29 \\
\hline $\mathrm{SE}(\mathrm{m})$ & 0.5 & 0.65 & 0.575 & 0.899 & 10.824 & 5.8615 \\
\hline $\mathrm{CV}$ & 0.786 & 1.011 & 0.8985 & 0.826 & 10.083 & 5.4545 \\
\hline
\end{tabular}


Table.6 Mean performance of parents, crosses and standard checks for ear height and ear girth in two seasons

\begin{tabular}{|c|c|c|c|c|c|c|}
\hline Source & $\begin{array}{c}\text { Rabi 2013-14 } \\
\text { ear height }(\mathrm{cm})\end{array}$ & $\begin{array}{c}\text { Kharif-2014 } \\
\text { ear height }(\mathrm{cm})\end{array}$ & $\begin{array}{l}\text { Mean } \\
(\mathrm{cm})\end{array}$ & $\begin{array}{l}\text { Rabi } 2013-14 \\
\text { cob girth }(\mathrm{cm})\end{array}$ & $\begin{array}{c}\text { Kharif } 2014 \\
\text { ear girth }(\mathrm{cm})\end{array}$ & $\begin{array}{l}\text { Mean } \\
(\mathrm{cm})\end{array}$ \\
\hline CB6-36 & 74.81 & 73.40 & 74.11 & 13.57 & 13.55 & 13.56 \\
\hline CB6-39 & 73.03 & 72.43 & 72.73 & 13.32 & 13.18 & 13.25 \\
\hline CB7-11 & 59.60 & 60.40 & 60.00 & 12.52 & 12.57 & 12.54 \\
\hline CB7-28 & 67.76 & 66.00 & 66.88 & 12.42 & 12.41 & 12.41 \\
\hline CB7-59 & 50.86 & 50.40 & 50.63 & 14.40 & 14.52 & 14.46 \\
\hline Lines mean & 65.21 & 64.53 & 64.87 & 13.25 & 13.25 & 13.24 \\
\hline Lines range & 50.86 to 74.81 & 50.40 to 73.40 & $\begin{array}{c}50.63 \text { to } \\
74.11\end{array}$ & 12.42 to 14.40 & 12.41 to 14.52 & 12.41 to 14.46 \\
\hline BML6 & 60.43 & 59.96 & 60.20 & 12.98 & 13.28 & 13.13 \\
\hline BML7 & 61.76 & 62.23 & 62.00 & 11.62 & 11.80 & 11.71 \\
\hline CML181 & 50.70 & 50.20 & 50.45 & 11.54 & 11.79 & 11.67 \\
\hline checks mean & 57.63 & 57.46 & 57.55 & 12.05 & 12.29 & 12.17 \\
\hline checks range & 50.70 to 61.76 & 50.20 to 62.23 & $\begin{array}{c}50.45 \text { to } \\
62.00\end{array}$ & 11.54 to 12.98 & 11.79 to 13.28 & 11.67 to 13.13 \\
\hline CB6-36×CB7-11 & 80.78 & 80.45 & 80.62 & 15.86 & 15.82 & 15.84 \\
\hline CB6-36×CB7-28 & 75.00 & 75.18 & 75.09 & 16.78 & 16.69 & 16.74 \\
\hline CB6-36×CB7-59 & 89.36 & 90.21 & 89.79 & 16.11 & 15.95 & 16.03 \\
\hline CB6-39×CB7-11 & 97.00 & 94.67 & 95.83 & 15.71 & 15.69 & 15.70 \\
\hline CB6-39×CB7-28 & 79.13 & 79.72 & 79.42 & 15.88 & 15.85 & 15.87 \\
\hline CB6-39×CB7-59 & 82.26 & 81.75 & 82.00 & 17.16 & 17.04 & 17.10 \\
\hline CB7-11×CB6-36 & 92.00 & 91.63 & 91.82 & 15.70 & 15.72 & 15.71 \\
\hline CB7-11×CB6-39 & 101.23 & 97.80 & 99.52 & 15.80 & 15.73 & 15.77 \\
\hline CB7-28×CB6-36 & 94.60 & 92.65 & 93.63 & 16.10 & 16.07 & 16.09 \\
\hline CB7-28×CB6-39 & 94.23 & 90.63 & 92.43 & 17.98 & 17.85 & 17.91 \\
\hline CB7-59×CB6-36 & 85.23 & 84.69 & 84.96 & 16.18 & 16.19 & 16.19 \\
\hline CB7-59×CB6-39 & 88.70 & 87.20 & 87.95 & 16.53 & 16.53 & 16.53 \\
\hline Hybrids mean & 88.29 & 87.22 & 87.76 & 16.32 & 16.26 & 16.29 \\
\hline Hybrids range & 75.00 to 101.23 & 75.18 to 97.80 & $\begin{array}{c}75.09 \text { to } \\
99.52\end{array}$ & 15.71 to 17.98 & 15.69 to 17.85 & 15.71 to 17.91 \\
\hline \multicolumn{7}{|l|}{ check } \\
\hline DHM-117 & 96.10 & 98.73 & 97.415 & 16.09 & 16.27 & 16.18 \\
\hline BML7×BML6 & 97.92 & 98.86 & 98.39 & 16.22 & 16.30 & 16.26 \\
\hline checks mean & 97.01 & 98.80 & 97.90 & 16.16 & 16.29 & 16.22 \\
\hline checkss range & 96.10 to 97.92 & 98.73 to 98.86 & $\begin{array}{c}97.41 \text { to } \\
98.39\end{array}$ & 16.09 to 16.22 & 16.27 to 16.30 & 16.18 to 16.26 \\
\hline $\mathrm{CD}$ & 1.681 & 2.398 & 2.0395 & 0.266 & 0.255 & 0.2605 \\
\hline $\mathrm{SE}(\mathrm{d})$ & 0.83 & 1.184 & 1.007 & 0.131 & 0.126 & 0.1285 \\
\hline SE (m) & 0.587 & 0.837 & 0.712 & 0.093 & 0.089 & 0.091 \\
\hline $\mathrm{CV}$ & 1.276 & 1.835 & 1.5555 & 1.07 & 1.026 & 1.048 \\
\hline
\end{tabular}


Table.7 Mean performance of parents, crosses and standard checks for ear length and number of kernel rows in two seasons

\begin{tabular}{|c|c|c|c|c|c|c|}
\hline Source & $\begin{array}{c}\text { Rabi 2013-14 } \\
\text { cob length } \\
(\mathrm{cm})\end{array}$ & $\begin{array}{c}\text { Kharif } 2014 \\
\text { ear length }(\mathrm{cm})\end{array}$ & Mean $(\mathrm{cm})$ & $\begin{array}{c}\text { Rabi 2013-14 } \\
\text { no of kernel } \\
\text { rows }\end{array}$ & $\begin{array}{c}\text { Kharif } 2014 \\
\text { no of kernel } \\
\text { rows }\end{array}$ & mean \\
\hline CB6-36 & 11.41 & 11.56 & 11.49 & 13.40 & 13.40 & 13.40 \\
\hline CB6-39 & 11.94 & 12.37 & 12.16 & 13.20 & 13.20 & 13.20 \\
\hline CB7-11 & 13.61 & 13.43 & 13.52 & 12.20 & 12.60 & 12.40 \\
\hline CB7-28 & 13.29 & 13.36 & 13.33 & 12.80 & 13.20 & 13.00 \\
\hline CB7-59 & 12.31 & 12.43 & 12.37 & 14.00 & 14.00 & 14.00 \\
\hline Lines mean & 12.51 & 12.63 & 12.57 & 13.12 & 13.28 & 13.20 \\
\hline Lines range & 11.41 to 13.61 & 11.56 to 13.43 & $\begin{array}{c}11.49 \text { to } \\
13.52\end{array}$ & 12.20 to 14.00 & 12.60 to 14.00 & 12.40 to 14.00 \\
\hline BML6 & 11.62 & 11.64 & 11.63 & 14.60 & 14.20 & 14.42 \\
\hline BML7 & 14.42 & 14.37 & 14.40 & 12.10 & 11.80 & 11.95 \\
\hline CML181 & 12.84 & 12.83 & 12.84 & 12.20 & 12.20 & 12.20 \\
\hline checks mean & 12.96 & 12.95 & 12.96 & 12.98 & 12.70 & 12.86 \\
\hline checks range & 11.62 to 14.42 & 11.64 to 14.37 & $\begin{array}{c}11.63 \text { to } \\
14.40\end{array}$ & 12.10 to 14.60 & 11.80 to 14.20 & 11.95 to 14.42 \\
\hline CB6-36×CB7-11 & 18.69 & 18.73 & 18.71 & 12.96 & 13.20 & 13.08 \\
\hline CB6-36×CB7-28 & 17.63 & 17.74 & 17.68 & 14.60 & 14.80 & 14.70 \\
\hline CB6-36×CB7-59 & 17.52 & 17.56 & 17.54 & 14.60 & 14.40 & 14.52 \\
\hline CB6-39×CB7-11 & 17.55 & 17.56 & 17.55 & 13.20 & 13.20 & 13.20 \\
\hline CB6-39×CB7-28 & 16.74 & 16.74 & 16.74 & 14.00 & 14.00 & 14.00 \\
\hline CB6-39×CB7-59 & 17.47 & 17.53 & 17.50 & 14.60 & 13.93 & 14.30 \\
\hline CB7-11×CB6-36 & 15.94 & 15.98 & 15.96 & 13.60 & 13.60 & 13.60 \\
\hline CB7-11×CB6-39 & 17.45 & 17.42 & 17.43 & 13.20 & 13.20 & 13.20 \\
\hline CB7-28×CB6-36 & 19.42 & 18.93 & 19.17 & 13.60 & 13.80 & 13.70 \\
\hline CB7-28×CB6-39 & 18.29 & 18.30 & 18.30 & 14.60 & 14.80 & 14.70 \\
\hline CB7-59×CB6-36 & 15.84 & 15.92 & 15.88 & 14.20 & 14.40 & 14.30 \\
\hline CB7-59×CB6-39 & 16.46 & 16.49 & 16.48 & 14.60 & 14.60 & 14.60 \\
\hline Hybrids mean & 17.42 & 17.41 & 17.41 & 13.99 & 13.99 & 13.99 \\
\hline Hybrids range & 15.84 to 19.42 & 15.98 to 18.93 & $\begin{array}{c}15.88 \text { to } \\
19.17\end{array}$ & 12.96 to 14.60 & 13.20 to 14.80 & 13.08 to 14.70 \\
\hline \multicolumn{7}{|l|}{ check } \\
\hline DHM-117 & 17.26 & 17.69 & 17.50 & 14.60 & 14.80 & 14.70 \\
\hline BML7×BML6 & 17.55 & 18.01 & 17.78 & 14.40 & 14.60 & 14.50 \\
\hline checks mean & 17.41 & 17.85 & 17.64 & 14.50 & 14.70 & 14.60 \\
\hline checks range & 17.26 to 17.55 & 17.69 to 18.01 & $\begin{array}{c}17.50 \text { to } \\
17.78\end{array}$ & 14.40 to 14.60 & 14.60 to 14.80 & 14.50 to 14.70 \\
\hline $\mathrm{CD}$ & 0.478 & 0.298 & 0.388 & 0.341 & 0.26 & 0.3005 \\
\hline $\mathrm{SE}(\mathrm{d})$ & 0.236 & 0.147 & 0.1915 & 0.168 & 0.129 & 0.1485 \\
\hline SE (m) & 0.167 & 0.104 & 0.1355 & 0.119 & 0.091 & 0.105 \\
\hline $\mathrm{CV}$ & 1.842 & 1.146 & 1.494 & 1.504 & 1.148 & 1.326 \\
\hline
\end{tabular}


Table.8 Mean performance of parents, crosses and standard checks for number of kernels/row and test weight in two seasons

\begin{tabular}{|c|c|c|c|c|c|c|}
\hline Source & $\begin{array}{c}\text { Rabi 2013-14 } \\
\text { no of kernels } \\
\text { /row }\end{array}$ & $\begin{array}{c}\text { Kharif } 2014 \\
\text { no of kernels } \\
\text { /row }\end{array}$ & mean & $\begin{array}{c}\text { Rabi 2013-14 } \\
100 \text { kernel wt } \\
\text { (g) }\end{array}$ & $\begin{array}{c}\text { Kharif } 2014 \\
100 \text { kernel wt } \\
\text { (g) }\end{array}$ & $\begin{array}{c}\text { Mean } \\
\text { (g) }\end{array}$ \\
\hline CB6-36 & 21.87 & 21.93 & 21.90 & 15.05 & 14.61 & 14.83 \\
\hline CB6-39 & 22.27 & 21.53 & 21.90 & 17.35 & 20.58 & 18.97 \\
\hline CB7-11 & 21.80 & 20.60 & 21.20 & 16.07 & 16.13 & 16.10 \\
\hline CB7-28 & 25.60 & 24.73 & 25.17 & 20.72 & 20.71 & 20.72 \\
\hline CB7-59 & 20.60 & 20.93 & 20.77 & 20.69 & 20.12 & 20.40 \\
\hline Lines mean & 22.43 & 21.94 & 22.19 & 17.98 & 18.43 & 18.20 \\
\hline Lines range & 20.60 to 25.60 & 20.60 to 24.73 & $\begin{array}{c}20.77 \text { to } \\
25.17\end{array}$ & 15.05 to 20.72 & 14.61 to 20.71 & 14.83 to 20.72 \\
\hline BML6 & 19.03 & 18.53 & 18.78 & 14.14 & 14.28 & 14.21 \\
\hline BML7 & 22.97 & 22.89 & 22.93 & 19.18 & 19.20 & 19.19 \\
\hline CML181 & 19.97 & 18.63 & 19.30 & 15.11 & 15.03 & 15.07 \\
\hline checks mean & 20.66 & 20.02 & 20.34 & 16.14 & 16.17 & 16.16 \\
\hline Checks range & 19.03 to 22.97 & 18.53 to 22.89 & $\begin{array}{c}18.78 \text { to } \\
22.93\end{array}$ & 14.14 to 19.18 & 14.28 to 19.20 & 14.21 to 19.19 \\
\hline CB6-36×CB7-11 & 40.50 & 38.73 & 39.62 & 31.43 & 31.93 & 31.68 \\
\hline CB6-36×CB7-28 & 37.60 & 36.93 & 37.27 & 33.53 & 33.65 & 33.59 \\
\hline CB6-36×CB7-59 & 38.83 & 39.00 & 38.92 & 32.28 & 32.66 & 32.47 \\
\hline CB6-39×CB7-11 & 38.40 & 37.67 & 38.03 & 33.06 & 33.19 & 33.13 \\
\hline CB6-39×CB7-28 & 39.53 & 39.57 & 39.55 & 27.21 & 27.12 & 27.16 \\
\hline CB6-39×CB7-59 & 37.76 & 37.77 & 37.76 & 28.60 & 28.13 & 28.36 \\
\hline CB7-11×CB6-36 & 36.13 & 37.03 & 36.58 & 32.11 & 32.24 & 32.17 \\
\hline CB7-11×CB6-39 & 38.00 & 36.59 & 37.30 & 33.84 & 33.71 & 33.78 \\
\hline CB7-28×CB6-36 & 39.20 & 39.83 & 39.52 & 33.06 & 33.28 & 33.17 \\
\hline CB7-28×CB6-39 & 36.60 & 36.83 & 36.72 & 34.23 & 33.68 & 33.95 \\
\hline CB7-59×CB6-36 & 34.70 & 35.09 & 34.90 & 34.68 & 34.40 & 34.54 \\
\hline CB7-59×CB6-39 & 34.23 & 36.13 & 35.18 & 33.28 & 33.22 & 33.25 \\
\hline Hybrids mean & 37.62 & 37.60 & 37.61 & 32.28 & 32.27 & 32.27 \\
\hline Hybrids range & 34.23 to 40.50 & 35.09 to 39.83 & $\begin{array}{c}34.90 \text { to } \\
39.55\end{array}$ & 27.21 to 34.68 & 27.12 to 34.40 & 27.16 to 34.54 \\
\hline \multicolumn{7}{|l|}{ check } \\
\hline DHM-117 & 36.67 & 38.15 & 37.41 & 33.88 & 33.51 & 33.70 \\
\hline BML7×BML6 & 38.60 & 38.63 & 38.615 & 33.72 & 34.13 & 33.90 \\
\hline checks mean & 37.64 & 38.39 & 38.01 & 33.80 & 33.82 & 33.80 \\
\hline checkss range & 36.67 to 38.60 & 38.15 to 38.63 & $\begin{array}{c}37.41 \text { to } \\
38.61\end{array}$ & 33.88 to 33.72 & 33.51 to 34.13 & 33.70 to 33.90 \\
\hline $\mathrm{CD}$ & 0.958 & 1.145 & 1.0515 & 2.182 & 0.612 & 1.397 \\
\hline $\mathrm{SE}(\mathrm{d})$ & 0.473 & 0.565 & 0.519 & 1.077 & 0.302 & 0.6895 \\
\hline SE (m) & 0.335 & 0.4 & 0.3675 & 0.762 & 0.214 & 0.488 \\
\hline $\mathrm{CV}$ & 1.819 & 2.183 & 2.001 & 4.893 & 1.367 & 3.13 \\
\hline
\end{tabular}


Table.9 Mean performance of parents, crosses and standard checks for yield/plant and tryptophan content in two seasons

\begin{tabular}{|c|c|c|c|c|c|c|}
\hline Source & $\begin{array}{l}\text { Rabi 2013-14 } \\
\text { yield/plant (g) }\end{array}$ & $\begin{array}{c}\text { Kharif } 2014 \\
\text { yield/plant } \\
\text { (g) }\end{array}$ & $\begin{array}{c}\text { Mean } \\
(\mathrm{g})\end{array}$ & $\begin{array}{c}\text { Rabi } 2013-14 \\
\text { tryptophan content } \\
(\%)\end{array}$ & $\begin{array}{l}\text { Kharif } 2014 \\
\text { tryptophan } \\
\text { content }(\%)\end{array}$ & $\begin{array}{c}\text { Mean } \\
(\%)\end{array}$ \\
\hline CB6-36 & 62.217 & 64.737 & 63.48 & 0.84 & 0.84 & 0.84 \\
\hline CB6-39 & 86.194 & 82.05 & 84.12 & 0.83 & 0.83 & 0.83 \\
\hline CB7-11 & 78.85 & 79.654 & 79.25 & 0.87 & 0.88 & 0.87 \\
\hline CB7-28 & 81.375 & 81.138 & 81.26 & 0.83 & 0.81 & 0.82 \\
\hline CB7-59 & 83.641 & 82.447 & 83.04 & 0.74 & 0.75 & 0.75 \\
\hline Lines mean & 78.46 & 78.01 & 78.23 & 0.82 & 0.82 & 0.82 \\
\hline Lines range & 62.22 to 86.19 & 64.73 to 82.44 & $\begin{array}{c}63.48 \text { to } \\
84.12\end{array}$ & 0.74 to 0.87 & 0.75 to 0.88 & 0.75 to 0.87 \\
\hline BML6 & 50.661 & 50.937 & 50.80 & 0.38 & 0.38 & 0.38 \\
\hline BML7 & 74.004 & 72.563 & 73.28 & 0.41 & 0.41 & 0.41 \\
\hline CML181 & 74.97 & 75.763 & 75.37 & 0.93 & 0.93 & 0.93 \\
\hline Checks mean & 66.55 & 66.42 & 66.48 & 0.57 & 0.57 & 0.57 \\
\hline Checks range & 50.66 to 74.97 & 50.93 to 75.76 & $\begin{array}{c}50.80 \text { to } \\
75.37\end{array}$ & 0.38 to 0.93 & 0.38 to 0.93 & 0.38 to 0.93 \\
\hline CB6-36×CB7-11 & 154.562 & 153.413 & 153.99 & 0.88 & 0.87 & 0.88 \\
\hline CB6-36×CB7-28 & 166.504 & 164.702 & 165.60 & 0.78 & 0.80 & 0.79 \\
\hline CB6-36×CB7-59 & 165.417 & 165.637 & 165.53 & 0.76 & 0.77 & 0.77 \\
\hline CB6-39×CB7-11 & 164.58 & 163.917 & 164.25 & 0.76 & 0.78 & 0.77 \\
\hline CB6-39×CB7-28 & 134.745 & 139.141 & 136.94 & 0.85 & 0.85 & 0.85 \\
\hline CB6-39×CB7-59 & 133.584 & 134.928 & 134.26 & 0.81 & 0.83 & 0.82 \\
\hline CB7-11×CB6-36 & 154.616 & 152.575 & 153.60 & 0.76 & 0.77 & 0.76 \\
\hline CB7-11×CB6-39 & 160.172 & 157.943 & 159.06 & 0.74 & 0.77 & 0.75 \\
\hline CB7-28×CB6-36 & 157.743 & 156.728 & 157.24 & 0.78 & 0.81 & 0.80 \\
\hline CB7-28×CB6-39 & 163.844 & 163.579 & 163.71 & 0.80 & 0.81 & 0.81 \\
\hline CB7-59×CB6-36 & 173.656 & 169.027 & 171.34 & 0.76 & 0.77 & 0.76 \\
\hline CB7-59×CB6-39 & 168.583 & 169.744 & 169.16 & 0.76 & 0.77 & 0.76 \\
\hline Hybrids mean & 158.17 & 157.61 & 157.89 & 0.79 & 0.80 & 0.79 \\
\hline Hybrids range & $\begin{array}{c}133.58 \text { to } \\
173.65\end{array}$ & 134.93 to 169.74 & $\begin{array}{c}134.26 \text { to } \\
171.34\end{array}$ & 0.74 to 0.88 & 0.77 to 0.87 & 0.75 to 0.88 \\
\hline \multicolumn{7}{|l|}{ check } \\
\hline DHM-117 & 162.423 & 161.85 & 162.14 & 0.52 & 0.52 & 0.52 \\
\hline BML7×BML6 & 171.18 & 169.65 & 170.42 & 0.51 & 0.52 & 0.51 \\
\hline checks mean & 166.80 & 165.75 & 166.28 & 0.52 & 0.52 & 0.52 \\
\hline checks range & $\begin{array}{c}162.42 \text { to } \\
171.18\end{array}$ & 161.85 to 169.65 & $\begin{array}{c}162.14 \text { to } \\
170.42\end{array}$ & 0.51 to 0.52 & 0.52 to 0.52 & 0.51 to 0.52 \\
\hline $\mathrm{CD}$ & 3.052 & 2.94 & 2.996 & 0.021 & 0.017 & 0.019 \\
\hline $\mathrm{SE}(\mathrm{d})$ & 1.507 & 1.452 & 1.4795 & 0.01 & 0.008 & 0.009 \\
\hline SE (m) & 1.066 & 1.027 & 1.0465 & 0.007 & 0.006 & 0.0065 \\
\hline $\mathrm{CV}$ & 1.438 & 0.391 & 0.9145 & 1.699 & 1.352 & 1.5255 \\
\hline
\end{tabular}


Fig.1 Comparison of normal DHM117 and QPM version of DHM 117 (BQPMH141) cobs

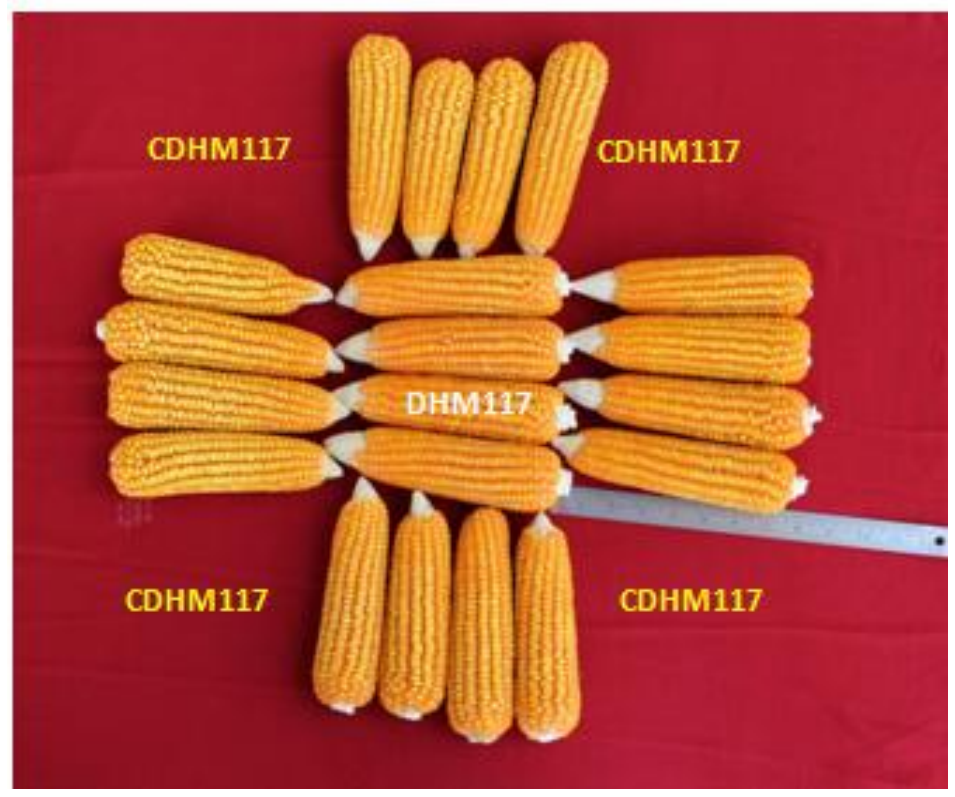

\section{Ear length (cm)}

The mean values for ear length ranged from 11.49 (CB6-36) to $13.52 \mathrm{~cm}$ (CB7-11) for converted lines, 11.63(BML 6) to $14.40 \mathrm{~cm}$ $(\mathrm{BML} 7)$ for testers and $15.88(\mathrm{CB} 7-59 \times$ CB6-36) to $19.17 \mathrm{~cm}(\mathrm{CB} 7-28 \times \mathrm{CB} 6-36)$ for hybrids. The lines recorded low mean for ear length (12.57) compared to testers (12.95) and hybrids (17.41). In case of checks, DHM 117 and reciprocal cross BML7 XBML6 recorded 17.47 and $17.78 \mathrm{~cm}$ respectively for ear length. Among the converted lines, CB7$11(13.52 \mathrm{~cm})$ recorded maximum ear length and CB7-28 was on par with it. Among the testers, BML7 $(14.40 \mathrm{~cm})$ was significantly superior to the remaining testers and converted lines (Table 4). Among the hybrids, maximum ear length was recorded for CB7$28 \times$ CB6-36 $(19.17 \mathrm{~cm})$ and other two hybrids (CB6-36 $\times$ CB7-11 and CB7-28 $\times$ CB6-36) were on par with the hybrid CB7-28 $\times$ CB6-36 and significantly superior over the other QPM hybrids. When compared to standard check DHM 117, six hybrids were significantly superior for ear length and remaining six hybrids were on par with it.

\section{Ear girth (cm)}

The mean values for ear girth ranged from 12.41(CB7-28) to $14.46 \mathrm{~cm}$ (CB7-59) for converted lines, 11.67 (CML181) to 13.13 (BML6) for testers and 15.71 (CB6-39 $\times$ CB7-11) to $17.91 \mathrm{~cm}(\mathrm{CB} 7-28 \times \mathrm{CB} 6-39)$ for QPM hybrids. The lines recorded high mean values for ear girth (13.24) compared to testers (12.17). In case of checks, DHM 117 and reciprocal cross BML7 $\times$ BML6 recorded 16.18 and $16.26 \mathrm{~cm}$ respectively for ear girth (Table 5).

Among the converted lines, CB7-59 (14.46 $\mathrm{cm})$ recorded maximum ear girth and was significantly superior to other lines. Among testers, BML6 $(13.13 \mathrm{~cm})$ was significantly superior to the remaining testers. The QPM hybrid CB7-28 $\times$ CB6-39 $(17.91 \mathrm{~cm})$ recorded maximum ear girth and two hybrids were on par to it and were significantly superior to the remaining hybrids. When compared to standard check DHM 117, three hybrids recorded significantly superior performance and remaining hybrids were on par with it. 


\section{Number of kernel rows per ear}

The mean values for number of kernel rows per ear ranged from $12.40(\mathrm{CB} 7-11)$ to 14.00 (CB759) for lines, 14.42 (BML6) to 11.95 (BML 7) for testers and $13.08(\mathrm{CB} 6-36 \times \mathrm{CB} 7-11)$ to $14.70(\mathrm{CB} 6-36 \times \mathrm{CB} 7-28$ and CB7-28 $\times$ CB639) for QPM hybrids. The converted lines recorded high mean values (13.07) compared to testers (12.86). In case of checks, DHM 117 and reciprocal cross BML7 $\times$ BML 6 recorded 14.70 and 14.50 number of kernel rows per ear respectively. Among lines, CB7-59 (14.00) recorded maximum number of kernel rows per ear and significantly superior to other converted lines. Among the testers, BML 6 (14.42) recorded maximum number of kernel rows per ear and CB7-59 was on par with it (Table 5). Among the hybrids, CB6-36 $\times$ CB7-28 and CB7-28 $\times$ CB6-39 recorded maximum number of kernel rows per ear and these two hybrids were on par with standard check. Over the standard check DHM 117 (14.70), two hybrids were on par to it for number of kernel rows per ear.

\section{Number of kernels per row}

Mean values for number of kernels per row ranged from 20.77 (CB7-59) to 25.17 (CB7-28) for converted lines, 18.78 (BML6) to 22.93 (BML7) for testers and 34.90 (CB7-59 × CB6$36)$ to $39.62(\mathrm{CB} 6-36 \times \mathrm{CB} 7-11)$ for hybrids. For number of kernels per row, the QPM hybrids recorded high mean values for number of kernels per row (37.61) compared to converted lines (22.19) and testers (20.34). In case of checks, DHM 117 and BML7 $\times$ BML6 recorded 37.41 and 38.61, respectively. Among the lines, CB7-28 (25.17) recorded maximum number of kernels per row and three lines on par with it (Table 6). Among the testers, BML 6 and CML181 were on par with BML7. Among the hybrids, two hybrids were on par with the best hybrid CB6-36 $\times$ CB7-11 and were significantly superior to the remaining hybrids. When compared with the standard check DHM 117 , only two QPM hybrids recorded significantly superior performance and remaining 10 hybrids were on par with it for number of kernels per row.

\section{0 kernel weight $(\mathrm{g})$}

Mean values for this trait ranged from 14.83 (CB6-36) to $20.72 \mathrm{~g}$ (CB7-28) for converted lines, 14.21 (BML6) to $19.19 \mathrm{~g}$ (BML7) for testers and 27.16 (CB6-39 $\times$ CB7-28) to $34.54 \mathrm{~g}$ (CB7-59 × CB6-36) for QPM hybrids. For 100 kernel weight, the hybrids recorded high mean values for 100 kernel weight (32.27 g) compared to converted lines $(18.20 \mathrm{~g})$ and testers $(16.16 \mathrm{~g})$. In case of standard checks, DHM 117 and BML7 $\times$ BML6 recorded 33.70 and $33.93 \mathrm{~g}$ mean values for 100 kernel weight, respectively. Among the converted lines, two lines were on par with the best line CB7-28. Among the testers, BML7 was significantly superior to the remaining testers. Among the QPM hybrids, CB7-28 × CB6-39 (33.95 g) was on par with the best hybrid CB7-59 $\times$ CB6-36 $(34.54 \mathrm{~g})$. When compared with the standard check DHM 117, ten hybrids were on par for 100 kernel weight but two hybrids recorded significantly superior mean values over DHM117.

\section{Grain yield per plant (g)}

The mean values for grain yield per plant ranged from 63.48 (CB6-36) to 84.12 g (CB639) for converted lines, 50.80 (BML6) to 75.37 $\mathrm{g}(\mathrm{CML} 181)$ for testers and 134.26 (CB6-39 $\times$ CB7-59) to $171.34 \mathrm{~g}(\mathrm{CB} 7-59 \times \mathrm{CB} 6-36)$ for QPM hybrids. Hybrids recorded high mean values for grain yield per plant $(157.89 \mathrm{~g})$ compared to lines (73.32 g) and testers (66.48 g). In case of checks, DHM 117 and reciprocal cross BML7 $\times$ BML6 recorded 162.14 and $170.41 \mathrm{~g}$ respectively (Table 7 ). Among the lines, CB7-59 (83.04g) was on par with the best line CB7-28 (84.12 g) and was significantly superior to the remaining lines. Among the testers, CML181 (75.37 g) was significantly superior to the remaining testers. Among the QPM hybrids, maximum grain yield per plant was recorded by CB7-59 $\times$ CB6-36 (171.34 g) and was significantly superior. When compared 
with the standard check DHM 117, six hybrids were on par but remaining six hybrids were recorded significantly superior mean values over DHM117. Maqsood et al., (2007), Rahman et al., (2010), Dragana et al., (2013) and Yusuf (2013) reported same kind of results.

\section{Tryptophan content (\%)}

The mean values for this character ranged from 0.75 (CB7-59) to 0.87 (CB7-11) for converted lines, 0.38 (BML6) to 0.93 (CML 181) for testers and $0.75(\mathrm{CB} 7-11 \times \mathrm{CB} 6-39)$ to 0.88 (CB6-36 × CB7-11) for hybrids. For kernel tryptophan content the converted lines recorded high mean values $(0.82 \%)$ than QPM hybrids (0.79) and testers (0.57). In case of checks, DHM 117 and reciprocal cross BML $7 \times$ BML 6 recorded 0.51 and $0.52 \%$ values respectively (Table 7). Among the lines, CB6-36 (0.84\%) was on par with the best line CB7-11 (0.87). Among the testers, CML181 (0.93\%) was significantly superior to the remaining testers and converted lines. Among the hybrids, maximum kernel tryptophan content was recorded by CB6-36 × CB7-11 (0.88). When compared with the standard check DHM 117, all hybrids were recorded significantly superior mean values over DHM 117. Earlier results reported by Bello et al., (2012) and Dragana et al., (2013) coincide with the present one.

The overall means of parental lines and their cross combinations revealed that the hybrids recorded superior performance than the parents with respect to plant height $(\mathrm{cm})$, ear height $(\mathrm{cm})$, number of kernel rows per ear, 100 kernel weight (g), grain yield per plant (g) and tryptophan content. Further, the hybrids in general were tall and high yielding over parents. The two cross combinations CB6-36 $\times$ CB7-28 and CB6-36 $\times$ CB7-59 exhibited relatively higher tryptophan content and on par grain yield per plant when compared to check DHM 117. Cross combination CB6-36 × CB7-28 and standard check DHM117 cob pictures was shown in Fig no 1. The hybrid combination CB6-36 $\times$ CB7-28 was shown excellent performance at AICROP on Maize trails during the year 2015-16 and the yield was $6924 \mathrm{KG} / \mathrm{ha}$ (BQPMH141) with higher tryptophan content $(0.83 \%)$ and these hybrid parental lines need to be further multiplied for commercial exploitation.

Cross combination CB6-36XCB7-28 recorded higher yield (170g/plant) on par with DHM117 hybrid with increased tryptophan $(0.78 \%)$ content. New QPM inbreds (CB6-36, CB6-30, CB6-39, CB7-11, CB7-28 and CB7-59) are useful for further breeding programs for development of QPM hybrids and yield was on par with normal DHM117.

The most important goal of QPM research has been to reduce malnutrition in target countries through direct human consumption, even though the impact as of now, has been great perceived. It is expected that greater impact will accrue out of development and dissemination of improved QPM hybrids worldwide.

The converted QPM inbreds (CBML6 and CBML7) developed in the present study provides an ideal platform for stacking number of nutritionally important traits such as enhanced $\mathrm{Fe}$ and $\mathrm{Zn}$, low phytate (for increased bioavailability of nutrients) and high provitamin A. Considering the pace and the technological developments in genomics and proteomics, molecular breeding will be most leading option in future for stacking nutritionally important traits in maize. Cross combination CB6-36 X CB7-28 recorded higher yield (170g/plant) which is on par with DHM117 hybrid with increased tryptophan $(0.78 \%)$ content. New QPM inbreds developed (CB6-36, CB6-30, CB6-39, CB7-11, CB7-28 and CB7-59) are useful for further breeding programs for development of improved QPM hybrids.

We further need to develop high throughput, low cost, easily accessible phenotying/ screening tools. Generating awareness among the society and building global and national partners for eliminating malnutrition will be future strengths for biofortified maize. Effective seed production and distribution systems, 
market strategies, strong partnership among research groups and need based government policies will help in solving the problems of many poor and undernourished people.

\section{Acknowledgements}

The work was supported by grants from Department of Biotechnology, Government of India. First author is thankful to UGC, Govt. of India for providing fellowship to pursue Ph.D. All authors are thankful to PJTSAU for providing facilities to conduct the research.

\section{References}

Bello O.B., J. Mahamood, M.S. Afolabi, M.A. Azeez, S.A. Ige, S.Y. Abdulmaliq and Oluleye F. 2012. Biochemical analysis and grain yield characteristics of quality protein maize (Zea mays L.) in the Southern Guinea Savanna of Nigeria. World Research Journal of Biochemistry. 1(1):11-19.

Dragana Ignjatovic-Micic, Marija Kostadinovic, Goran Stankovic, Ksenija Markovic, Jelena Vancetovic, Sofija Bozinovic, Violeta Andjelkovic. 2013. Biochemical and agronomic performance of quality protein maize hybrids adapted to temperate regions. Maydica 58:311-317.

Fisher, R.A and Yates, F. 1967. Statistical tables for Biological, Agricultural and Medical Research, Longmen Group Limited. London.

Gupta, H.S., Babu, R., Agrawal, P.K., Mahajan, V., Hossain, F and Thirunavukkarasu, N. 2013. Accelerated development of quality protein maize hybrid through markerassisted introgression of opaque 2 allele.
Plant Breeding. 132: 77-82.

Krivanek, A.F., H. De Groote and Gunaratna, N.S. 2007. Breeding and disseminating quality protein maize (QPM) for Africa. African Journal of Biotechnology. 6: 312324.

Maqsood Qamar, Zulfiqar Ali Gurmani, Haq Nawaz Malik and Sikander Khan Tanveer. 2007. Evaluation of maize hybrids/synthetics under double cropping zone of northern areas of Pakistan. Sarhad J. Agric. 23(4).1009-1012.

Mbuya, K., K.K. Nkongolo, and KalonjiMbuyi, A. 2011. Nutritional analysis of Quality Protein Maize Varieties selected for agronomic characteristics in a breeding program. International Journal of Plant Breeding and Genetics. 5(4): 317-327.

Panse, V.G., and Sukhatme, P.V. 1985. Statistical methods for Agricultural workers, Indian Council of Agricultural Research, New Delhi.

Rahman, H., Durreshawar, S. Ali, F. Iftikhar, I.H. Khalil, S.M.A. Shah and Ahmad H. 2010. Stability analysis of maize hybrids across North West of Pakistan. Pak. J. Bot., 42(2): 1083-1091.

Sofi, P.A., W.A. Shafiq, A.G. Rather and Shabir, W.H. 2009. Review article: Quality protein maize (QPM): Genetic manipulation for the nutritional fortification of maize. Journal of Plant Breeding and Crop Science. 1 (6):244253.

Yusuf, M. 2013 Performance of single cross quality protein maize hybrids evaluated at Samaru-northern guinea savanna zone of Nigeria. Journal of Plant Breeding and Crop Science. 5(12): 239-244.

\section{How to cite this article:}

Surender, M., Deepti Sagare, Prashant Setti, Ch.V. Durga Rani, Farzana Jabeen, M.R. Sudarshan and Sokka Reddy, S. 2017. Mean Performance of Normal and QPM Maize Genotypes for Yield and Tryptophan Content. Int.J.Curr.Microbiol.App.Sci. 6(11): 830-844. doi: https://doi.org/10.20546/ijcmas.2017.611.098 